\title{
Welcome to the splice age: antisense oligonucleotide- mediated exon skipping gains wider applicability
}

\author{
Elizabeth M. McNally and Eugene J. Wyatt \\ Center for Genetic Medicine, Northwestern University, Chicago, Illinois, USA.
}

\begin{abstract}
Exon skipping uses antisense oligonucleotides (ASOs) to alter transcript splicing for the purpose of rescuing or modulating protein expression. In this issue of the $J C I$, Lee and colleagues developed and evaluated an ASO-dependent method for treating certain molecularly defined diseases associated with alterations in lamin A/C (LMNA) splicing. Exon skipping by ASOs is gaining traction as a therapeutic strategy, and the use of ASOs is now being applied to bypass mutations and generate modified but functional proteins for an array of genetic disorders.
\end{abstract}

\section{Exon skipping and neuromuscular disease}

There has been considerable progress toward using exon skipping for therapeutic intent for inherited muscle diseases in humans. Significant efforts have focused on such strategies for treating Duchenne muscular dystrophy (DMD), a lethal $\mathrm{X}$-linked recessive disorder caused by mutations in the gene encoding dystrophin (reviewed in ref. 1). In the case of $\mathrm{DMD}$, exon skipping is applied to convert out-of-frame mutations that ablate protein expression to those mutations that restore the open reading frame, thereby converting severe disease to milder disease. A primary antisense oligonucleotide (ASO) strategy in clinical development targets exon 51 of the dystrophin gene, as exon deletions proximal to this region are a relatively common cause of DMD. In the setting of these specific exon deletions, ASO-mediated exclusion of exon 51 generates in-frame mature RNA transcripts and rescues protein expression. The protein encoded by the ASO-modified transcript results in a relatively small internal deletion of the dystrophin protein (2).

\section{Moving ASOs beyond DMD}

With progress, ASO exon skipping approaches are now moving beyond the original DMD paradigm. Strategies for exon skipping have been developed for a number of neuromuscular conditions, including limb girdle muscular dystrophy (3), Pompe disease (4), cardiomyopathies $(5,6)$, and cystic fibrosis (7). Other approaches toward treating laminopathies have also been tested (8). For DMD, approaches extend beyond correction of the dystrophin gene and include combining ASO exon skipping with targeted reduction of myostatin $(9,10)$. ASO-induced exon skipping may be useful beyond rare genetic diseases, extending to disorders like rheumatoid arthritis (11) and cancer (12).

\section{A strategy to treat laminopathies}

In this issue, Lee and colleagues piloted an ASO-directed approach to treat Hutchinson Gilford progeria syndrome (HGPS) (13), a rare disorder that is often associated with a de novo dominant mutation in the lamin $\mathrm{A} / \mathrm{C}(L M N A)$ gene that disrupts the processing of lamin A (14). Lamin A and lamin $C$ are produced from a single gene

Related Article: p. 1592

Conflict of interest: E.M. McNally and E.J. Wyatt are coinventors on a patent related to exon skipping for limb girdle muscular dystrophy.

Reference information: / Clin Invest. 2016;126(4):1236-1238. doi:10.1172/JCI86799.

through alternative splicing and together form the core filamentous structure that lines the inner nuclear membrane (Figure 1). Lamin $A$ is initially produced as prelamin A, which includes an additional 98 amino acids at the carboxy terminus. Embedded in the last four amino acids of prelamin A are signals that direct farnesylation, which is linked to cleavage and processing to help localize mature lamin $\mathrm{A}$ at the inner nuclear membrane. Lamin $\mathrm{C}$ is encoded by an alternative splice form that terminates in exon 10 and does not undergo this maturation process. The de novo HGPScausing mutation (c.1824 C>T) disrupts splicing and leads to excess accumulation of prelamin A, also called progerin.

Lee et al. designed and tested ASOs to shift the balance of splicing to encode less lamin A and more lamin C (13). The rationale for this shift was justified by previous observations in lamin C-only mice, which, by genetic design, do not express lamin A in favor of lamin C (15). Despite the loss of lamin A, lamin C-only mice have no discernible phenotype, suggesting that changing the lamin $\mathrm{A} / \mathrm{C}$ ratio toward lamin $C$ could be an effective strategy to treat HGPS and other LMNA-related diseases. Mutations in LMNA produce a wide array of genetic diseases. The most common LMNA mutant phenotypes induce myopathic processes that affect the heart and skeletal muscle (16); however, LMNA mutations also cause lipodystrophyrelated phenotypes, bony malformation syndromes, and the premature aging phenotype seen in HGPS (16). Notably, the majority of LMNA mutant-related phenotypes are dominant; therefore, any ASO-targeted approach must specifically target the mutant allele and not the WT allele. Given that most mutations in LMNA are single nucleotide changes, it has proven challenging to design ASOs that preferentially target the mutant alleles. In such cases, off-target effects that affect the normal allele could result in knock down of the entire gene product, leading 
A

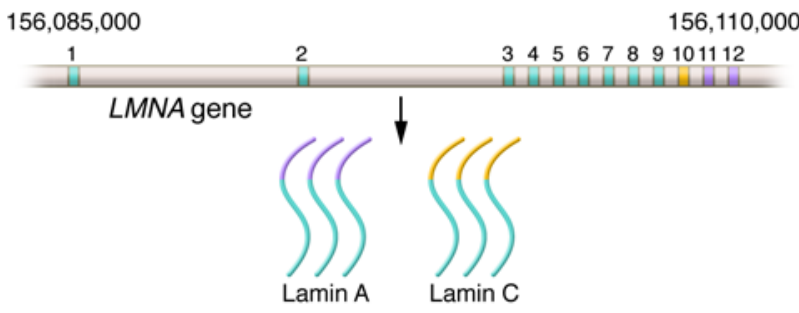

Normal lamin $A / C$ ratio
B

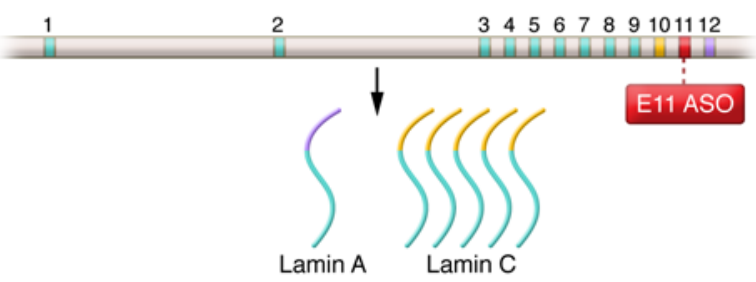

ASO-induced decrease of lamin $\mathrm{A} / \mathrm{C}$ ratio

Figure 1. A schematic of the LMNA gene, which spans approximately 25 kbp on chromosome 1 and encodes lamin A and lamin C. (A) Lamin A and lamin $C$ are produced from a single gene through alternative splicing. (B) Laminopathies result from mutations that alter lamin A processing. ASOs were designed to target exon 11 to shift the ratio of lamin A-encoding mRNA to lamin C-encoding mRNA. This strategy would treat laminopathies due to mutations in exon 11 and 12. Exons 1-12 are shown.

to unwanted toxicity. To circumnavigate this potential side effect, Lee et al. used an approach that could alter both mutant and WT alleles by targeting exon 11 sequences.

The effect of exon 11 ASO-mediated targeting was tested in murine and human fibroblasts (13). This approach increased lamin C expression and partially rescued the HGPS phenotype in vitro. HGPS cells were similarly exposed to exon 11 ASOs to confirm that ASO-induced exon skipping did not inadvertently increase prelamin A levels. Further investigation showed that exon splice enhancer-binding sequences are conserved between mouse exon 11 and human exon 11. Moreover, Lee et al. demonstrated a role for serine/argininerich splicing factor 2 (SRSF2, also known as SC35) in the regulation of LMNA splicing (13). ASO-mediated exon skipping was evaluated in a novel mouse model that carries the HGPS-associated G609G mutation. Based on observed outcomes in a previously generated transgenic model of HGPS, the most reliable disease phenotype in this new progeria mouse model is the loss of smooth muscle cells in a variety of tissues (17). Vascular abnormalities are a key aspect of the HGPS disease process, and treatment of G609G-expressing mice with exon 11 ASOs reduced adventitial fibrosis and improved vascular smooth muscle cell numbers. Together, these findings support the use of exon skipping to treat HGPS and to potentially treat any $L M N A$-associated disease that results from mutations in exon 11 or 12 .

\section{The ASO strategy}

The use of molecular correction to treat disorders like muscular dystrophies or laminopathies is challenged by the need to deliver systemic therapy and to do so with targeted efficacy. A potential attraction of ASOs is their comparative ease of delivery, as ASOs are small compounds that can be delivered subcutaneously or intravenously to achieve a systemic effect. While oral delivery has not yet been possible, systemic delivery is. Among ASOs, there are cell- and tissue-type differences in uptake, and cell penetration varies with ASO chemistry. The safety and efficacy of ASO-directed treatment is currently being evaluated in humans in phase I and II clinical trials for DMD (18), myotonic dystrophy (NCT02312011), and spinal muscular atrophy (NCT02193074). With this progress, the field is now turning to evaluating ASOs for potential utility in treating both genetic and nongenetic disorders.

\section{Old and new chemistry for ASOs}

ASO chemistry is evolving, and newer modifications are designed to improve ASO pharmacokinetics. Considerable effort has gone into modifying nucleotides to improve their target affinity, ability to penetrate cells, systemic stability, and resistance to degradation by nucleases. Distinct from RNA approaches directed at silencing mutant alleles, ASOs for exon skipping are designed to avoid RNase $\mathrm{H}$-mediated RNA degradation, thereby preserving a mature, modified, proteincoding transcript. Two common chemistries used for exon-skipping ASOs include 2'-O-methyl phosphorothioate (referred to as $2^{\prime} \mathrm{OMePS}$ ) and phosphorodiamidate morpholino oligomer (referred to as PMO). The negative charges on the phosphoro- thioate linkages in $2^{\prime} \mathrm{OMePS}$ may prolong ASO half life and differ from the neutrally charged PMO, although the negatively charged moieties are thought to interact with TLRs, potentially triggering a greater inflammatory response (reviewed in ref. 2). Recently, tricyclo-DNA-modified ASO compounds have been tested in the $m d x$ model for DMD and have been demonstrated to have better penetration into the heart and brain (19). Newer chemistries are emerging, and these are anticipated to improve ASO potency through affinity as well as a reduced side effect profile (20).

\section{The splice age}

As we move into the era of precise molecular correction, there are considerable limitations in moving these therapies into humans (21). In many instances, ASOmediated exon skipping is only able to target subgroups of individuals with rare diseases, often requiring precise sequences designed to treat personalized diseases. Current regulatory procedures are not well suited to evaluate this type of rare disease treatment, and the path forward may require modification of the regulatory process. Treatment with ASOs is also expected to require lifelong administration and be costly, another significant barrier to implementing ASO exon skipping as a treatment for disease. As we look to the horizon, gene editing using CRISPR/Cas9 may develop based on similar principles to ASO-induced exon skipping and similarly may require precise treatment design for individual patients. Indeed, three recent studies have shown that such genome editing is able to at least partially restore dystrophin expression and improve skel- 
etal muscle function in dystrophic mice (22-24). While this offers tremendous promise as curative strategy for genetic disease, many hurdles exist before human clinical implementation. Thus, utilization of ASOs may prove to be an efficient bridge between current therapeutic options and the future of genetic medicine at the molecular, therapeutic, and regulatory levels. Welcome to the splice age.

\section{Acknowledgments}

This work was supported by NIH grant R01 HL061322.

Address correspondence to: Elizabeth M. McNally, 303 E. Superior St., Center for Genetic Medicine, Northwestern University Feinberg School of Medicine, Chicago, Illinois 60611, USA. Phone: 312. 503.5600; E-mail: Elizabeth.mcnally@ northwestern.edu.

1. Crunkhorn S. Muscular dystrophy: New exon-skipping strategy rescues dystrophin. Nat Rev Drug Discov. 2015;14(4):236.

2. Kole R, Krieg AM. Exon skipping therapy for Duchenne muscular dystrophy. Adv Drug Deliv Rev. 2015;87:104-107.

3. Gao QQ, et al. Reengineering a transmembrane protein to treat muscular dystrophy using exon skipping. J Clin Invest. 2015;125(11):4186-4195.

4. Clayton NP, et al. Antisense oligonucleotidemediated suppression of muscle glycogen synthase 1 synthesis as an approach for substrate reduction therapy of Pompe disease. $\mathrm{Mol}$ Ther Nucleic Acids. 2014;3:e206.

5. Gedicke-Hornung C, et al. Rescue of cardiomyopathy through U7snRNA-mediated exon skipping in Mybpc3-targeted knock-in mice. EMBO Mol Med. 2013;5(7):1060-1077.

6. Gramlich M, et al. Antisense-mediated exon skipping: a therapeutic strategy for titin-based dilated cardiomyopathy. EMBO Mol Med. 2015;7(5):562-576.

7. Igreja S, Clarke LA, Botelho HM, Marques L, Amaral MD. Correction of a cystic fibrosis splicing mutation by antisense oligonucleotides. Hum Mutat. 2016;37(2):209-215.

8. Scharner J, Figeac N, Ellis JA, Zammit PS. Ameliorating pathogenesis by removing an exon containing a missense mutation: a potential exon-skipping therapy for laminopathies. Gene Ther. 2015;22(6):503-515.

9. Lu-Nguyen NB, Jarmin SA, Saleh AF, Popplewell L, Gait MJ, Dickson G. Combination antisense treatment for destructive exon skipping of myostatin and open reading frame rescue of dystrophin in neonatal mdx mice. Mol Ther. 2015;23(8):1341-1348.

10. Kemaladewi DU, et al. Dual exon skipping in myostatin and dystrophin for Duchenne muscular dystrophy. BMC Med Genomics. 2011;4:36.

11. Yilmaz-Elis S, et al. Antisense oligonucleotide mediated exon skipping as a potential strategy for the treatment of a variety of inflammatory diseases such as rheumatoid arthritis. Ann Rheum Dis. 2012;71(suppl 2):i75-i77.

12. Dewaele M, et al. Antisense oligonucleotidemediated MDM4 exon 6 skipping impairs tumor growth. JClin Invest. 2016;126(1):68-84.

13. Lee JM, et al. Modulation of $L M N A$ splicing as a strategy to treat prelamin A diseases. JClin Invest. 2016;126(4):1592-1602.

14. Eriksson M, et al. Recurrent de novo point mutations in lamin A cause HutchinsonGilford progeria syndrome. Nature. 2003; 423(6937):293-298.

15. Fong LG, et al. Prelamin A and lamin A appear to be dispensable in the nuclear lamina. JClin Invest. 2006;116(3):743-752.

16. Cattin ME, Muchir A, Bonne G. 'State-of-theheart' of cardiac laminopathies. Curr Opin Cardiol. 2013;28(3):297-304

17. Varga R, et al. Progressive vascular smooth muscle cell defects in a mouse model of Hutchinson-Gilford progeria syndrome. Proc Natl Acad Sci U S A. 2006;103(9):3250-3255.

18. Voit $\mathrm{T}$, et al. Safety and efficacy of drisapersen for the treatment of Duchenne muscular dystrophy (DEMAND II): an exploratory, randomised, placebo-controlled phase 2 study. Lancet Neurol. 2014;13(10):987-996.

19. Goyenvalle A, et al. Functional correction in mouse models of muscular dystrophy using exon-skipping tricyclo-DNA oligomers. Nat Med. 2015;21(3):270-275.

20. Jarver P, O’Donovan L, Gait MJ. A chemical view of oligonucleotides for exon skipping and related drug applications. Nucleic Acid Ther. 2014;24(1):37-47.

21. Aartsma-Rus A, et al. Translational and regulatory challenges for exon skipping therapies. Hum Gene Ther. 2014;25(10):885-892.

22. Long C, et al. Postnatal genome editing partially restores dystrophin expression in a mouse model of muscular dystrophy. Science. 2016;351(6271):400-403.

23. Nelson CE, et al. In vivo genome editing improves muscle function in a mouse model of Duchenne muscular dystrophy. Science. 2016;351(6271):403-407.

24. Tabebordbar M, et al. In vivo gene editing in dystrophic mouse muscle and muscle stem cells. Science. 2016;351(6271):407-411. 